\title{
Pluralism without Genic Causes?*
}

\author{
Elisabeth A. Lloyd, Matthew Dunn, Jennifer Cianciollo, \\ and Costas Mannouris $\$$
}

Since the fundamental challenge that I laid at the doorstep of the pluralists was to defend, with nonderivative models, a strong notion of genic cause, it is fatal that Waters has failed to meet that challenge. Waters agrees with me that there is only a single cause operating in these models, but he argues for a notion of causal 'parsing' to sustain the viability of some form of pluralism. Waters and his colleagues have some very interesting and important ideas about the sciences, involving pluralism and parsing or partitioning causes, but they are ideas in search of an example. He thinks he has found an example in the case of hierarchical and genic selection. I think he has not.

\section{Methodological and Epistemological Concerns.}

1.1. Introductory Points. Since the fundamental challenge that I laid at the doorstep of the pluralists was to defend, with reasons or nonderivative models, a strong notion of genic cause, it is fatal that Waters has failed to meet that challenge. He gives no new arguments for the existence of independent genic causes. Despite my arguments that there is no sense to be made of the notion of independent genic causal models, Waters continues to refer to genic causes. Waters appears to agree with me that there is only a single cause operating in these models, but he argues for a notion of causal 'parsing' to sustain the viability of some form of pluralism. Nevertheless, the fundamental point is that he seems to have explicitly abandoned the notion of an independent genic cause. My analysis in Section 6 of "Why the Gene Will Not Return" (Lloyd 2005) stands.

*Received May 2005.

$\dagger$ To contact the authors, please write to: Elisabeth A. Lloyd, Department of History and Philosophy of Science, 1011 East Third Street, Goodbody Hall 130, Indiana University, Bloomington, IN 47405; e-mail: ealloyd@indiana.edu.

\$We would like to thank Jason Baker, Stephen Crowley, Melinda Fagan, Patrick Forber, and Michael Weisberg for their extensive comments and discussion. We are indebted to Ken Waters for his generous assistance and friendship throughout the production of these papers.

Philosophy of Science, 72 (April 2005) pp. 334-341. 0031-8248/2005/7202-0003\$10.00

Copyright 2005 by the Philosophy of Science Association. All rights reserved. 
While that is really most of what there is to be said, and that is selfevident from reading Waters' paper, there are several other points raised by Waters that deserve to be addressed.

First, a minor point. Waters seems to imply that I made up the term, 'genic pluralism'. Not so. S\&K ${ }^{1}$ (1988) use it to describe their own position, which I assumed that Waters was also defending in KSW (1990). I understand that he wants to distance himself from such a position, and I welcome his retraction of claims made in that paper that seem to indicate a preference for the genic view. Waters admits that pragmatic considerations disfavor the use of genic models, and favor the multilevel modelsunder the assumption that these models are empirically adequate.

Secondly, Waters seems to think that he and his coauthors hold a monopoly on the term 'pluralism'. But, I discussed three different types of pluralism in my target paper-a circumstance that he completely ignores. Waters continues to speak as if his type of pluralism is the only type, rather than acknowledging the type of pluralist I actually am. His view is unduly restrictive.

1.2. History, Method, and Merit. One standard objection to my position is winningly put by Waters: "It is not as if the diploid model owns the information above the alleles and the genic model owns the information below the diploid genotype" (Waters 2005, 321; emphasis added). The key point here is the priority of actual discovery. When a factorfor example, a level of interactor-has been discovered to have an influence on the outcome of a selective process via the application of a hierarchical model or method, it seems only reasonable to say that the hierarchical approach should be credited with that discovery. If the model is later translated into a genic model, and the genic advocates then complain that the hierarchists don't own the higher-level information, well . . . in a sense that's true . . . but here they seem to be insensitive to the empirical side of the biology.

It is a historical fact that the investigators of the sickle-cell gene used a diploid model (Taliaferro and Huck 1923; Neel 1947). It is a historical fact that the discovers of the full selection regime of the $t$-allele used a gametic model, a diploid model, and a demic model (Lewontin and Dunn 1990; Lewontin 1962). Therefore, it is a historical fact that the genic versions of these models are derivative.

But, philosophers will tell us that it is irrelevant which model was involved in producing the actual higher-level information. In other words, what is important, according to Waters, is that it is possible to describe 
causality differently in the two different sorts of model — genic and hierarchical - and that both of these sorts of models draw upon multiple levels of information. The latter claim is true; the former, I do not grant. But again, and it is a scientific and empirical question, Where and how do we obtain the multiple levels of information that are used in these models?

It might appear to be Waters' hidden assumption in this argument that philosophy of science is not concerned with the empirical value of actual scientific theories, but only with their metaphysical implications. In other words, he seems unconcerned about which approach has yielded the crucial information required to advance the field. On my view, however, if philosophy of science is weighing the value of the genic and hierarchical approaches, then surely their empirical merits (particularly their epistemological and methodological strengths) must be included in such a judgment.

So, suppose we were unaware of higher-level information and its impact on evolutionary outcomes. Using the genic approach, how would we obtain such information? Famously, George Williams advocated a rule of thumb for answering this question: Do not look for higher-level information at all unless the genic level has been proven to be empirically inadequate. Equally famously, the vast majority of practicing geneticists and behavioral biologists took this recommendation as a dogmatic law (see Lloyd [1988] 1994, 95, 121-122). Thus, it was the hierarchical selectionists - and only the hierarchical selectionists - who successfully formulated the methods for seeking out and documenting the higher-level interactions that were involved in evolutionary dynamics involving kin groups, demes, etc. (Lloyd 2005). In contrast, Waters proposes that "[g]enic selection theoreticians learn that a genic environment needs to be partitioned in a model, if there are different selective pressures on the allele type in different spaces of the genic environment" (Waters 2005, 322). In other words, he needs to partition the environment, just in case partitioning the environment makes a difference to gene fitness. And how will a genic theorist know this?

Waters proposes that the genic selectionists are not just capable of accommodating, or representing truthfully, but also seeking out such higher-level interactions. Has he given any reasons for thinking that this is so? Is there any evidence to support this claim? Is there an example of genic selectionists successfully establishing a higher-level causal force where hierarchical selectionists missed it? No, no, and no.

In sum, 'ownership' of the higher-level information does not rest on simple historical priority. Rather, methodologically, the hierarchical views have developed approaches and methods for seeking out higher-level information (and they have been very effective at finding it), while the genic 
approaches have no such methods, making them epistemically and methodologically inferior. Moreover, they have a history of methodological dogmatism opposing the very existence of such methods. Furthermore, Waters has given us no reason to believe that genic models hold any promise of grounding practices designed to obtain such information, without simply borrowing the methods already formulated for hierarchical models. Finally, all of this is highly relevant, because philosophical evaluation of the merits of the genic and hierarchical approaches demands consideration of their methodological and epistemological value, not only of their causal or metaphysical standing.

Certainly, Waters does not wish to return us to pre-Kuhnian days wherein we sanitize our pure metaphysical questions about units of selection from details of the actual practice and history of the science. Nothing in his body of work would lead me to think so. Given that I have argued in my target paper that the genic model construction and metaphysical conclusions are bound together in the arguments as presented, my opponents are not free to slice off metaphysical questions as they wish.

Still, we can expect that Waters and sympathizers will respond that the genic view is on a causal par with the hierarchical view, and that the documented methodological and epistemic priority or superiority of the hierarchical view cuts no ice metaphysically. I must remind the reader, then, that this argument has already been thoroughly addressed in Section 6 of "Why the Gene will Not Return." I claim that the separation of metaphysical from epistemological and methodological issues is not sustainable, unless the argument there is rebutted.

\subsection{Derivation. Finally, let me note one final, odd claim. Waters writes:}

Of course, the genic model is going to include information about causal interactions in the environment. The fact that it depends on such information doesn't make it derivative of the diploid-genotypic model. $(2005,321)$

Can Waters mean to claim that the genic model is not derivative? $\mathrm{He}$ can't mean this. It is straightforwardly mathematically and logically derivative. It is also straightforwardly historically derivative. So, we could say, it is both 'timelessly' and historically derivative. Is there some other form of 'derivative' that Waters has in mind?

The issue of derivation is crucial. A derived model can contain nothing that the model from which it was derived did not also contain. The impact of this is that there cannot be any new causal or metaphysical information in the derived model. But since the issue of the causal independence of 
these models has provoked such confusion, let's consider the arguments for such independence in more detail.

\section{Causes, Multiple Models, and Metaphysics.}

2.1. Alternate Models. Waters asserts that I disagree with the statement that there are cases where models do not disagree about facts $(2005,312)$. Another way to put this point is that there are alternate models for the same situation.

I do not disagree with this. I never have. If Waters and his supporters read Chapter 4 of my book, The Structure and Confirmation of Evolutionary Theory ([1988] 1994), they will find discussion and analysis of such cases.

Another key point concerns the prevalence of what Waters calls PERM situations. In practicing population genetics, ${ }^{2}$ this actually involves a confined set of models, contrary to the impression the reader might get from Waters' paper. More importantly, there are a number of important empirical and theoretical cases from hierarchical selection that are not PERM cases, including the $t$-allele case featured in Waters' original 'Tempered Realism" (1991). (Cf. Goodnight and Stevens 1997; Wade 1985.) Since Waters denies that PERM cases involve different causes, but involve only different causal 'parsings', it becomes imperative to understand precisely what this latter term means. Remember, in order to sustain a substantial form of metaphysical pluralism, the different models were originally claimed to represent distinct causes, including genic causes. In Section 6 of the target paper, I argue that without a distinct and independent genic causal level, there are no genuinely independent models being represented, and therefore, no real alternatives among which to choose. Now, Waters proposes replacing such 'real' causes with 'parsed' causes. He also wishes to replace the metaphysical pluralism of $\mathrm{S} \& \mathrm{~K}$ with an 'epistemological' pluralism. Our focus, then, will be on whether such parsed causes can possibly supply interestingly independent explanations from among which to choose. I turn now to the section written by my colleagues.

2.2. Parsing. ${ }^{3}$ Waters argues that we are compelled to accept pluralism because two or more different models can be constructed that represent the same selection process equally well. He notes that in some selection processes the conceptual division between selected domains and environments can be drawn in at least two different ways, both being correct in

2. See Waters' citations of Dugatkin and Reeve 1994; Kerr and Godfrey-Smith 2002.

3. This section is written by Matthew Dunn, Jennifer Cianciolo, and Costas Mannouris. 
that they result in empirically adequate models. By drawing different conceptual divisions between selected domains and environments, Waters claims that the models are 'parsing the causes' involved in the selection process differently, i.e., each parsing captures the same underlying causal interactions. He argues that the existence of two empirically adequate models of the same selection process that parse the causes differently is good reason to adopt a form of pluralism about levels of selection - to admit that there is not always a "single, correct way to represent the causal structure of the world" $(2005,312)$.

Waters operationalizes the notion of 'parsing causes' by redrawing the conceptual division between selected domain and environment. One such redrawing might involve simply 'changing the labels'. For example, 'causal interplay in the environment' in one model becomes 'interactors involved in a selection process' in the other. But, this does not support an interesting pluralism; it is simply substituting synonyms.

At the other end of the spectrum, a strong pluralism would result if two empirically adequate models of the same selection process represented different causal interactions. For example, in the sickle-cell case, if $W_{S}^{A} \neq W_{S A}$ when both models adequately account for the frequency of the sickle allele, this would be an interesting pluralism, because the two models would be representing different underlying causal processes. But, this is not the case. And importantly, as we have seen, Waters does not require that there be a pluralism of underlying causal structures. Moreover, in the sickle-cell case, Waters explicitly endorses the view that the genic and diploid-genotype models identify the same underlying causal structure.

Waters' account of 'parsing causes' must therefore fall somewhere between the weak pluralism of relabeling and the strong pluralism of different causal interactions. The most charitable rendering of this notion would seem to be something like the following. In genic accounts, the boundary between the environmental domain and the selected domain is drawn between two alleles at a single locus. In the sickle-cell case, the diploid-genotype model does not draw any causally relevant, conceptual distinction between these two alleles; therefore, the difference between the two models is not simply one of relabeling. Yet, the two models contain exactly the same information about causal interactions - this information is simply partitioned differently. It is in this sense, then, that Waters is a pluralist. There are different ways to partition the same information in models that account for the same underlying causes equally well. We choose among parsings based on pragmatic considerations, which may have important consequences, as spelled out by Waters' discussion (2005) of Reichenbach on volitional decisions.

The key point, though, remains that the genic and genotypic models 
represent the same causal structure. Waters owes us an account of how, given that Lloyd has argued that both of these model types are derivative from one another, they could represent substantively different causal parsings. As things stand, they do not seem able to capture any interesting or robust form of pluralism.

2.3. Genic Causes. The other new addition in Waters' paper is the application of Woodward's theory of the manipulability notion of causes to the genic case. This appeal, like that to parsing, will not recover a robust notion of genic cause for Waters. This is because Waters has misrepresented my position, here. He claims that I am a monist, and that I think the diploid causal model gives the only true cause, while the genic causal model does not. This is false. The truth is that I don't think there is any interesting, independent genic causal model at all. And Waters-even using Woodward's very valuable and sensible theory of causality-has failed to address the challenges laid down in Section 6 and Section 8 of the target paper, regarding agency and tractability in a genic theory.

Fundamentally, Waters is trying to use parsing to get a notion of genic causality off the ground, by casting genes as interactors. But, I argued at length in my paper - an argument that Waters has not rebutted - that genic selectionists fail in that effort. In sum, when we attempt to operationalize Waters' notion of parsing, we end up with a weak view that cannot support the kind of distinctions necessary to make a real difference in the theoretical choices available to us.

In general, allelic models can be (and often are) used as simplifying tools, guides to research, hints about good modeling techniques, and calculating devices (Michod 1982; Dugatkin and Reeve 1994; Kerr and Godfrey-Smith 2002). But, allelic models have never been shown to sustain a notion of genic cause that is not derivative, and nothing in Waters' present paper successfully shows otherwise.

2.4. Waters' Pluralism. This raises the issue of what Waters is really doing with his whole project. He is, as he says, interested in a fundamental epistemological issue, involving cases in which there are multiple models of causation - each of which is true, but none of which is uniquely true. I would like the reader to entertain a possibility: Waters and his colleagues have some very interesting and important ideas about the sciences, involving pluralism and parsing or partitioning causes, but they are ideas in search of an example. He thinks he has found an example in the case of hierarchical and genic selection. I think he has not. 


\section{REFERENCES}

Dugatkin, Lee Allan, and Hudson Kern Reeve (1994), "Behavioral Ecology and Levels of Selection: Dissolving the Group Selection Controversy", Advances in the Study of Behavior 23: 101-133.

Goodnight, Charles, and Lori Stevens (1997), "Experimental Studies of Group Selection: What Do They Tell Us about Group Selection in Nature?", American Naturalist 150: S59-S79.

Kerr, Benjamin, and Peter Godfrey-Smith (2002), "Individualist and Multilevel Perspectives on Selection in Structured Populations", Biology and Philosophy 17: 477-517.

Kitcher, Philip, Kim Sterelny, and Kenneth Waters (1990), "The Illusory Riches of Sober's Monism”, Journal of Philosophy 87: 158-160.

Lewontin, Richard (1962), "Interdeme Selection Controlling a Polymorphism in the House Mouse", American Naturalist 96: 65-78.

Lewontin, Richard, and Leslie Clarence Dunn (1960), "The Evolutionary Dynamics of a Polymorphism in the House Mouse", Genetics 45: 705-722.

Lloyd, Elisabeth A. ([1988] 1994), The Structure and Confirmation of Evolutionary Theory. Reprint. Originally published by Greenwood Press. Princeton, NJ: Princeton University Press.

(2005), "Why the Gene Will Not Return", Philosophy of Science 72: 287-310.

Michod, Richard (1982), "The Theory of Kin Selection", Annual Review of Ecology and Systematics 13: 23-55.

Neel, James (1947), "The Clinical Detection of the Genetic Carriers of Inherited Disease", Medicine 26: 115-153.

Sterelny, Kim, and Philip Kitcher (1988), "The Return of the Gene", Journal of Philosophy 85: $339-361$.

Taliaferro, William, and John Huck (1923), "The Inheritance of Sickle-Cell Anemia in Man", Genetics 8: 594-598.

Wade, Michael (1985), "Soft Selection, Hard Selection, Kin Selection, and Group Selection", American Naturalist 125: 61-73.

Waters, C. Kenneth (1991), "Tempered Realism about the Force of Selection", Philosophy of Science 58: 553-573.

(2005), "Why Genic and Multilevel Selection Theories Are Here to Stay", Philosophy of Science 72: 311-333. 


\section{Erratum}

"Pluralism without Genic Causes?" (in vol. 72, no. 2, April 2005) contains a misspelling of a coauthor's surname. The correct spelling is 'Cianciolo'.

Philosophy of Science, 72 (July 2005) p. 529. 0031-8248/2005/7203-0020\$10.00

Copyright 2005 by the Philosophy of Science Association. All rights reserved. 